\title{
Short and Long Term Results of Major Lung Resections in Very Elderly People
}

\author{
Cristian Rapicetta, Massimiliano Paci, Tommaso Ricchetti, \\ Sara Tenconi, Salvatore De Franco and Giorgio Sgarbi
}

Additional information is available at the end of the chapter

http://dx.doi.org/10.5772/50987

\section{Introduction}

Improvement in health care has lead to increased life expectancy through the chronicization of formerly fatal diseases. On the other hand, increasing duration of exposure to risk factors means that the incidence of lung cancer increases in proportion to age, and is actually the first cause of death for malignancy in the world.

Consequently, a growing number of very elderly people, aged 80 or more, are presenting with a resectable lung tumour. The increased prevalence of cardiovascular and pulmonary comorbidity and consequently poor performance status often make patients unfit to surgery, which remains the treatment of choice for early stages Non-Small Cell Lung Cancer (NSCLC) [1, 2]. Increasing age is often associated with lower likelihood of treatment and more liberal use of radiation therapy than of surgery [3].

\subsection{Background}

In a recent paper Janssen and Kunst found that in 2050 predicted life expectancy for people reaching 80 years will be 9.16 years for men and 12.65 for women [4], which clearly overcome the prognosis of lung cancer. Therefore, cancer-related mortality will likely have a even more great impact on octogenarians survival.

Although several studies have shown that long term survival after surgery can be achieved in a significant proportion of octogenarians. However the minimal prognosis of untreated or palliated early stage lung cancer (1.5 years) forces to go beyond palliative cares [5].

Recently, even more Authors reported results after surgery in very elderly patients: unfortunately many studies consist of retrospective, non-randomized studies on small number of patients making difficult to define risk factors for complications and mortality. 
Considering the improvement in preoperative functional assessment, anaesthetic care, pain relief, postoperative facilities and modern surgical techniques, it is possible that more octogenarians could be successfully operated, but an accurate selection of patients is mandatory to avoid excessively high incidence of complications and mortality. Moreover long term results such as respiratory function and quality of life (QOL) is as well as important in decision making process to schedule or deny surgery: even less data are available in this field, particularly comparing surgery to medical therapies (chemo- and radiotherapy).

Established that surgery is undoubtedly the best therapy for early stage lung cancer to date, especially in terms of local control of disease, this chapter aims to analyse short (complications, mortality) and long-term (respiratory function) of very elderly people. Analysing results in a large cohort of patients and reviewing literature we try to suggest proper selection criteria to surgery in these patients, considering risk/benefit of alternative treatment modalities.

\section{Report of a single institution experience}

My colleagues and I reported some years ago our results in a large series of octogenarians consecutively operated on between January 1990 through December 2005 [6]. Life expectancy in the province is greater than the national average and octogenarians have always represented a significant percentage of patients with resectable lung cancer.

\subsection{Material and methods}

The lung cancer database of a single centre (Thoracic Surgery Unit at the University Hospital of Siena) was retrospectively reviewed to identify all patients aged $>=80$ years who underwent lung resection for NSCLC with curative intent in the period considered above. We tried to limit the period of study to 1990, mainly because the very few octogenarians treated before 90 's and secondly to eliminate bias due to patients selection, clinical staging and functional assessment through decades.

All patients were extensive evaluated throughout medical history, physical examination, routine blood tests, electrocardiogram, echocardiography, blood gas analysis, spirometry and calculation of diffusion capacity of the lung for carbon monoxide (DLCO). Second level tests were performed when indicated: cardio-respiratory test and/or lung perfusion scintigraphy in case of poor pulmonary function at basal spirometry (predicted postoperative FEV1\% less than $40 \%$, computed on the basis of pulmonary segments to be removed); cardiac stress tests and eventually coronary angiography were performed in presence of clinical symptoms of angina pectoris, significant ischemic signs at basal electrocardiogram, or segmental cardiac discinesia at basal echocardiography.

Clinical staging was based on chest CT-scan, bronchoscopy, CT-scan of chest, abdomen and brain and bone scintigraphy. Mediastinoscopy was electively performed in case of enlarged mediastinal lymphnodes at CT scan (short axis $>1 \mathrm{~cm}$ ) with irregular pattern of postcontrast perfusion. PET was not routinely used due to unavailability in the hospital of Siena 
but was performed in the last seven consecutive patients resulting positive only in the site of primary tumour. Whenever possible, a preoperative diagnosis was done, even by transpleural methods (Fine Needle trans-thoracic Aspiration - FNA).

Indication to surgery was established on the basis of features of disease (determining resectability), and features of the patient (determining operability). Efforts spent to achieve accurate preoperative staging were finalized to select early clinical stages (I-II). Operability of the patients was judged in a multidisciplinary setting including pneumologist and anaesthesiologist. Decision was based on functional status and comorbidities: we did not considered an age-limit as a contraindication per se to surgery. Our surgical policy favoured lobectomies over sublobar resections and whenever possible we tried to avoid pneumonectomy. Sublobar resection was preferred by the attending surgeon in case of poor respiratory function and/or severe cardiovascular comorbidities, and was considered a compromise: anatomical segmentectomies were however preferred to wedge resections and were considered adequate for stage I peripherally located tumours.

None of the patients received neoadjuvant therapy for at least two reasons: first, patients in advanced clinical stages (III or IV) were denied to surgery in favour of low dose chemo or radiation therapy and best supportive care; second, postoperative risk of major lung resection after induction chemotherapy was judged excessively high in such patients

Adjuvant therapy was administered in case of unforeseen lymph nodal disease or residual disease. Radiotherapy was preferred, although 4 patients in very good health conditions could tolerate chemotherapy.

Postoperative complications were classified as minor (non life-threatening) or mayor (potentially life-threatening), according to gravity of the disease and its mortality in literature and in our population.

Data recorded included demographic and clinical features of population (gender, age, smoking history, performance status, preoperative pulmonary function tests, comorbidities, use of induction therapies), as well as perioperative data (type of lung resection, operative mortality - defined as death within 30 days from surgery or during the same hospitalization, complications, length of in-hospital stay, admission to ICU, histology, pathologic stage and the use of adjuvant therapy.

Follow up included total-body CT scan and serum cancer markers every 6 months for first 2 years, then chest CT scan every 12 months until the fifth years. Bronchoscopy and recently, PET-scan were performed only in case of suspected local or distance recurrent disease.

Deaths included all causes but we differentiated only cancer and non-cancer related deaths.

Binary logistic regression analysis was used to discriminate independent risk factors for mayor complications and 30-days mortality after surgical resection. Long term survival curves were computed by the Kaplan-Meier method and compared with Log-rank test. Cox regression analysis was used to determine significance of prognostic factors for long term survival. 


\subsection{Results}

In the period considered, 96 octogenarians affected by NSCLC underwent surgery with curative intent ( $5.6 \%$ of 1711 lung resections performed during the same period).

Over the years an increase of percentage of octogenarians having a pulmonary resection for NSCLC was observed (from $2.7 \%$ in 1990 to $14 \%$ in 2005): it reflects the considerations expressed above and it is likely that there will be a growing trend of such percentages in next years.

Population was predominantly male (84), with age ranging from 80 to 89 (median, 82 years).

Data of population is shown in Table I: some of the data are worthy of note: almost all patients $(90 \%)$ had a history of cigarette smoking with 40 median pack-years and almost half of them $(48.9 \%)$ presented comorbidities, mainly cardiopulmonary. Thirteen patients had a history of coronary artery disease, defined as a history of prior myocardial infarction, prior coronary stenting or by-pass or angina pectoris. Despite this percentages, almost all the octogenarians (94-97.9\%) were Eastern Cooperative Oncology Group (ECOG) status 0 or 1.

The surgical procedures performed included 59 standard lobectomies, 4 extended lobectomies, (one upper sleeve lobectomy, 3 en block chest wall resections), 13 wedge resections ( 2 performed by VATS), 9 pneumonectomies, 7 segmentectomies and 4 bilobectomies. Patients with $\mathrm{pO} 2<65 \mathrm{mmHg}$ or a pCO2 $>45 \mathrm{mmHg}$ and/or MVV or an FEV1< $50 \%$ of predicted were scheduled for sublobar resection.

The histological examination of the resected specimens revealed NSCLC in all patients: one of the aims of preoperative histological biopsy was to exclude histology with proven poor survival (small-cell carcinoma).

Regarding pathologic stage, $61(63,5 \%)$ patients were confirmed to be in stage IA or IB: concordance with clinical staging was not so high but it should be taken into account unavailability of modern diagnostic tools for staging. Adjuvant chemotherapy was administered in 6 patients and radiotherapy in 2 patients due to unforeseen pathological N2 disease or unexpected residual microscopic disease.

One or more complications developed in 42 patients for a total morbidity of $44 \%$. Five patients suffered multiple complications. Mayor complications lead to death 9 of 17 patients (52.9\%) resulting in an overall mortality rate of $9.4 \%$ (Table 2 ).

The median postoperative hospitalization was 8 days, not significantly longer than in younger patients (7 days) although 7 patients were discharged after more than 2 weeks. Eighty patients $(83 \%)$ were discharged home without need of further rehabilitation; only 10 patients $(9.6 \%)$ were discharged to a convalescent care facility.

It must be underlined that no patient suffered permanent or prolonged disability after lung resection although post-thoracotomy pain syndrome was present in a small percentage after several months (14\% after 1 year), causing significant discomfort. 
Mean age (years)

$\operatorname{Sex}(\mathrm{M} / \mathrm{F})$

Smokers

Comorbidities

$>1$ comorbidity

Respiratory parameters

FEV1 (\% of predicted)

CO diffusion (\% of predicted)

$\mathrm{PaO} 2(\mathrm{~mm} \mathrm{Hg})$

Pathological stage

I

II

III*

IV§

Surgical procedure

Lobectomy

Sublobar resection

Pneumonectomy

Extended resection

Bilobectomy

En-bloc resection with chest wall

Histology

Adenocarcinoma

Squamous cell carcinoma

Adenosquamous carcinoma

Large cell carcinoma

Comorbidities

Coronary artery disease*

Chronic obstructive pulmonary disease

Vascular disease

Diabetes mellitus

Chronic atrial fibrillation

Heart valve disease

Kidney failure
$81.5 \pm 1$

$84 / 12$

$86(90 \%)$

47 (49\%)

$13(13.5 \%)$

$75.8 \% \pm 16.2 \%$

$76.5 \% \pm 20.7 \%$

$75.1 \pm 11.4$

$61(63.5 \%)$

17 (17.7\%)

$17(17.7 \%)$

1 (1\%)

$68(70.8 \%)$

$19(19.8 \%)$

$9(9.4 \%)$

$4(4.2 \%)$

$3(3.1 \%)$

42 (43.8\%)

48 (50\%)

$2(2.1 \%)$

$4(4.2 \%)$

13 (13.5\%)

19 (19.7\%)

13 (13.5\%)

$8(8.3 \%)$

4 (4.1\%)

$3(3.1 \%)$

$1(1 \%)$

$\mathrm{FEV}_{1}=$ forced expiratory volume in $1 \mathrm{~s}$.

* N2 disease in 12 cases and T3N1 disease in 5 cases $\S$ contralateral pulmonary metastasis

Table 1. Demographic, clinical, and pathological data of patients 


\begin{tabular}{lcc}
\hline \multicolumn{1}{c}{ Major complications } & N. (\%) & 30-day mortality (\%) \\
\hline Respiratory failure & 3 & 3 \\
ARDS & 3 & 2 \\
Broncho-pleural fistula & 2 & 1 \\
Cardiac failure & 2 & \\
Kidney failure & 1 & 1 \\
Pneumonia & 1 & 1 \\
Pulmonary embolism & 1 & \\
Stroke & 1 & \\
Pulmonary edema & 1 & 1 \\
Hematemesis & 1 & $9(9)$ \\
IMA & 1 & \\
\hline
\end{tabular}

Table 2. Major complications rate and mortality

Using binary logistic regression analysis, among the pre- and peri-operative covariates analyzed, we found that resection of more than 1 lobe $(p=0.008)$, cardio-respiratory comorbidity ( $\mathrm{p}=0.042), \mathrm{PaO} 2<75 \mathrm{mmHg}(\mathrm{p}=0.029)$ and $\mathrm{DLCO}<60 \%(\mathrm{p}=0.023)$ were predictive of major complications with the first risk factor predicting 30-day mortality too $(\mathrm{p}=0.01)$. Detailed results are reported in Table 3 and 4 .

\begin{tabular}{lccc} 
Covariate & $\mathrm{RR}$ & $95 \% \mathrm{CI}$ & $p$ \\
\hline Extended resection & $\mathbf{1 0 . 5}$ & $\mathbf{1 . 9 - 5 9 . 7}$ & $\mathbf{0 . 0 0 8}$ \\
Comorbidity & $\mathbf{5}$ & $\mathbf{1 . 0 6 - 2 4}$ & $\mathbf{0 . 0 4 2}$ \\
$\mathrm{FEV}_{1}<60 \%$ & 0.45 & $0.08-2.5$ & 0.36 \\
$\mathrm{CO} \mathrm{diffusion}^{*} \mathbf{6 0} \%$ & $\mathbf{6 . 2 4}$ & $\mathbf{1 . 2 8}-\mathbf{3 0 . 3 7}$ & $\mathbf{0 . 0 2 3}$ \\
$\mathrm{PaO}_{2}<75 \mathrm{~mm} \mathrm{Hg}$ & $\mathbf{5 . 4 2}$ & $\mathbf{1 . 1 9 - 2 4 . 6 5}$ & $\mathbf{0 . 0 2 9}$ \\
$\mathrm{Age}$ & 0.7 & $0.46-1.04$ & 0.076 \\
$\mathrm{Sex}$ & 0.17 & $0.014-2.21$ & 0.18 \\
\hline
\end{tabular}

*Bilobectomy, pneumonectomy, or en-bloc chest wall resection. $\mathrm{CI}=$ confidence interval, $\mathrm{FEV}_{1}=$ forced expiratory volume in $1 \mathrm{~s}, \mathrm{RR}=$ relative risk.

Table 3. Predictors of major postoperative complications

\begin{tabular}{lccc} 
Covariate Value & $\mathrm{RR}$ & $95 \% \mathrm{CI}$ & $p$ \\
\hline Extended resection & $\mathbf{1 2 . 9 6}$ & $\mathbf{1 . 8 5 - 9 0 . 8 9}$ & $\mathbf{0 . 0 1}$ \\
Comorbidity & 6.69 & $0.69-64.69$ & 0.1 \\
$\mathrm{FEV}_{1}<60 \%$ & 1.59 & $0.2-12.87$ & 0.66 \\
$\mathrm{CO} \mathrm{diffusion}<60 \%$ & 3.97 & $0.52-30.61$ & 0.18 \\
$\mathrm{PaO}_{2}<75 \mathrm{~mm} \mathrm{Hg}$ & 1.08 & $0.18-6.36$ & 0.93 \\
$\mathrm{Age}$ & 0.78 & $0.47-1.31$ & 0.36 \\
Sex & 1.15 & $0.08-17.36$ & 0.91 \\
\hline
\end{tabular}

Table 4. Predictors of 30-day postoperative mortality 
At the mean follow-up of 42 months (range 8 months to 12 years), 46 (47.9\%) patients were alive and 50 (52\%) had died.

It was surprising that, despite the advanced age, the majority of patients (29/50, 71\%) died of cancer, either by local recurrence or distant metastasis.

The 5-year survival rate was significantly different between stage I and stage III (60\%vs14\%, $\mathrm{p}<0.001)$ and between stage II and stage III $(42 \% v s 14 \%, \mathrm{p}=0.046)$. There was no statistical difference in 5-year survival rate between stage I and stage II ( $\mathrm{p}=0.39)$. The overall 3- and 5year survival rates were $51 \%$ and $34 \%$, respectively, with a median survival of 3.7 years (95\% CI, 2.7 to 4.6 years) (Figure 1 ).

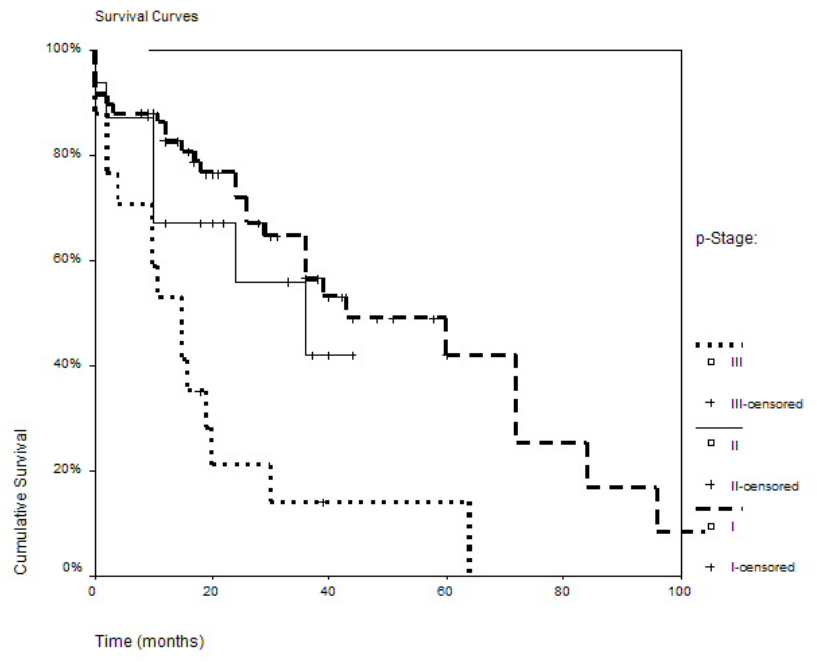

Figure 1. Actuarial survival according to pathological stage.

Using the Cox proportional hazard model of multivariate analysis, only pathological stage of disease resulted as an independent predictor of long term survival $(p=0.003)$ : stage II or more carries a relative risk of 4.2 to die of disease (Table 5).

\begin{tabular}{lccc} 
Covariate & $\mathrm{RR}$ & $95 \% \mathrm{CI}$ & $p$ \\
\hline Pathological stage (II-III Vs I) & $\mathbf{4 . 2}$ & $\mathbf{1 . 7 4 - 1 0 . 4 6}$ & $\mathbf{0 . 0 0 1}$ \\
Extended resection & 1.2 & $0.37-3.6$ & 0.81 \\
Comorbidity & 16.4 & $1.1-237.5$ & 0.38 \\
$\mathrm{FEV}_{1}$ & 1 & $0.97-1.03$ & 0.87 \\
$\mathrm{DLCO}$ & 1 & $0.98-1.02$ & 0.56 \\
$\mathrm{PaO}_{2}$ & 0.95 & $0.95-1.2$ & 0.295 \\
$\mathrm{Age}$ & 0.97 & $0.79-1.2$ & 0.81 \\
Sex & 0.61 & $0.17-2.2$ & 0.44 \\
\hline
\end{tabular}

Table 5. Predictors of long-term survival 


\subsection{Interpretation of results}

Although recent studies have demonstrated acceptable long term survival after lung cancer surgery in octogenarians $[7,8,9,10]$, elderly patients are less likely to have surgical resection [4], because advanced age is an important risk factor for postoperative complications and death, [1, 2]. In fact, despite an improvement in early outcome in the past ten years, the risk of life-threatening complications for octogenarians remains variable: Harvey et al., reported a $1.6 \%$ risk in patients $70-79$ years old compared to a $17.6 \%$ rate in octogenarians [11] and Naunheim et al. [12] reported that even within the octogenarians, increasing age was a negative factor.

The $9.4 \%$ operative mortality observed in this series is similar to other reports covering the same time period [13] but was exceedingly higher in patients who required an extended resection (more than one lobe or chest wall and lung en bloc resection), as found also by other Authors. [14, 15].

The mortality rate after pneumonectomy and even after bilobectomy was remarkable: $22 \%$ and $25 \%$, respectively: it is well known that operative mortality is substantial in elderly people having extended resection, especially right sided pneumonectomy, with mortality exceeding $20 \%[1,16,17]$. Besides, the British Thoracic Society noted that pneumonectomy is associated with a higher mortality risk in the elderly and that age should be a factor in deciding suitability for pneumonectomy.

These results convinced us to consider for surgical operation only octogenarians with lung cancers amenable to be resected by lobectomy. In addition, also pulmonary resections combined with en bloc chest wall removal should be avoided since they carry an increased mortality rate as previously reported by $[2,18]$. The mortality rate was instead acceptable after standard lobectomy $(8.4 \%$, result in line with those reported in literature) and was null after wedge resection or segmentectomy.

We observed 17 major complications (mainly cardiopulmonary) in 16 patients, and is note worthy that they lead to death in more than half of the cases $(9 / 17,52.6 \%)$.

Cardiac complications developed frequently during the postoperative period. All patients had an echocardiogram but exercise stress testing was only executed in case of symptomatic angina, history of infarction or ECG abnormalities. The efficacy and cost-effectiveness of exercise stress testing or dipyridamole thallium scan as screening tests is controversial, but probably we should use a lower threshold for these second- and third-level investigations in the octogenarians. When they result positive, it is useful to proceed to coronary angiography because patients found to have significant coronary lesions amenable to angioplasty, can be successfully operated on after 2 months (3 cases in our experience). On the other hand, if coronary artery bypass surgery would be required, we prefer to manage the patient nonoperatively with radiotherapy, because we believe that combined heart-lung surgery carries an excessive risk in advanced age people [19]. 
Long term survival is also of critical importance in this patient population with limited natural life expectancy.

Our overall 5-year survival of 35\% is consistent with that reported in similar series over the past decade, with 5 -year survival rates ranging from $16 \%$ to $55 \%[7,8,9,10,20,21]$.

As expected pathologic stage was an independent prognostic factor for long-term survival but, differently from previous studies in the octogenarians except for one [14], our survival curves justify surgery also for patients with stage II NSCLC, with 5-year survival rate of $42 \%$. Survival in stage III disease is uniformly very poor in all the series and is significantly lower than in the younger counterparts, probably because a fewer percentage of older patients are able to tolerate extended surgical procedures and complete multimodal treatments. A recent report of Fanucchi and coll. reported a low 5-years survival: Authors correctly recognized that it was due to unexpected high incidence of stage III disease because of inaccurate clinical staging [22].

The QOL and the level of independence after surgery should be a major component in the decision-making process in the elderly.

We did not identify any prolonged disability and the fact that nearly $80 \%$ of octogenarians in this cohort were discharged directly to home suggests that most patients were able to resume the preoperative lifestyle.

\section{Lung volume reduction era and its importance in lung cancer surgery}

In the late 1950s, Brantigan and co-workers were the first to present the modern concept of lung reduction for emphysema, theorizing that by surgically reducing lung volume, part of circumferential pull upon the bronchioles could be restored, diaphragm could be brought higher with more efficient function and size of the thoracic cage could be reduced, allowing for better contraction of the intercostal muscles and ultimately reducing airflow obstruction and relieving dyspnoea [23]. Further results confirmed efficacy of lung volume reduction surgery (LVRS), with outcome improving parallel to refinement of selection criteria, although long-term survival did not seem to be positively affected as symptoms and pulmonary function.

Because lung cancer and emphysema share common risk factors, it is not surprising to find that both conditions are often seen in the same individual as stated in the text. First papers addressing combined adequate cancer resection with volume reduction were published only in 1998 and were based on very small series of patients (5 and 14): all of them did well postoperatively and showed long term benefits in dyspnoea index, FEV1, and 6-minute walk test $[24,25]$.

In the evaluation of patients candidate for surgery, spirometry is still considered the gold standard: ppoFEV1\% is the most powerful predictor of medical postoperative mortality and morbidity as demonstrated by Kearney and coll. [26]. The second-level assessments as inhalation-perfusion lung scintigraphy and cardio-respiratory exercise test may be useful in 
further evaluation of "critical" patients: however, while they demonstrated valid predictors of postoperative complications, they did not clearly show a higher accuracy than simple ppoFEV1\% obtained by calculation of broncho-pulmonary segments removed [27].

McKenna and coll. [28] reported in 1997 that improvement in poFEV1\% and dyspnoea score was more evident in patients aged under 70, although the differences were not statistical significant. A growing number of studies in the recent years have reported an improvement of respiratory function after lobectomy in some patients affected by COPD [29], or at least a minimal impairment of respiratory function indicated by better apoFEV\% (actual postoperative) than ppoFEV1\% (predicted postoperative)[30]. In such cases Authors have hypothesized that lobectomy could have a lung volume reduction surgery (LVRS) effect [31].

This data from literature suggested us that even elderly patients with less severe COPD could get some benefit from resection of emphysematous parenchyma and therefore in the decision making process that lead to schedule an octogenarian to lung surgery, additional considerations should be made in evaluating respiratory function: not only preoperative FEV1\% but also ppoFEV1\% could be inaccurate in some cases in predicting the real postsurgical pulmonary function loss. Moreover, other studies reported that abnormal pulmonary function (FEV1\%<60\%) was not associated with mortality $[20,7,10]$, with only first study reporting higher incidence of complications.

Curative surgery chance to octogenarians affected by emphysema and lung cancer is therefore to be seriously considered.

\section{Long term respiratory function in our series}

A previous study conducted Thoracic Surgery Unit at University Hospital of Siena on patients who underwent pneumonectomy demonstrated that a lower preoperative respiratory function was associated to a much lower loss after pneumonectomy [32]: these data, together with some comforting results of bilateral LVRS procedures performed by VATS in the same Institution, stimulated us to analyzed late variation of respiratory parameters in the same cohort of octogenarians presented above. Results were published in 2011 [33].

\subsection{Materials and methods}

In order to analyze a subgroup as more homogeneous as possible, we considered only patients with COPD (defined by a Tiffenau index $<0.7$ ). Since sublobar resections demonstrated to be well tolerated by elderly patients and resection of more than 1 lobe or combined chest wall resection is not recommended (being associated to major complications and in-hospital mortality) we limited analysis to standard lobectomy. Patients who received adjuvant therapies (anyway a small number) and those who continued smoking were excluded in order to eliminate bias. Spirometry was not routinely repeated at predetermined time and it was decided to consider patients with spirometry executed after $12 \pm 3$ months 
from surgery (in absence of local relapsing disease or exacerbation of COPD). Pulmonary Function Test (PFT) were performed after bronchodilator inhalation in order to eliminate the reversible component of airflow impairment Out of the 96 patients 24 fulfilled all inclusion criteria and formed the population of the study.

Obviously removing non-functioning lung tissue does not affect pulmonary reserve, however no patients presented atelectasis or obstructive pneumonia of lobe to be resected.

Predicted Postoperative FEV1\% (ppoFEV1\%) was computed not simply on the basis of crude amount of segments to be resected: the attenuation value of lung parenchyma (between -600 and -900 Houmsfield Unit) was used to compute the percentage of functioning tissue resected and to correct the predicted-postoperative FEV1, as proposed by WU et al [Wu 1994]. This method had been demonstrated to be a valid substitute of lung perfusion scintigraphy, with a good correlation of values found by the two methods [Yoshimoto 2009]; however in patients with worst respiratory impairment (FEV1<60\%) lung perfusion scintigraphy was executed and used to compute ppoFEV1.

Roentgenographic pattern of emphysema (homogeneous Vs upper lobe) was established according to criteria proposed by Goddard and coll. for CT-scan [34]; data were analyzed using unpaired Student t-test for comparison of means of continuous variables between groups, paired t-test was used to assess differences within groups between preoperative and postoperative values.

\subsection{Results}

Patients were categorized into two groups (Group 1: moderate COPD; Group 2: mild COPD) according to the Global initiative for chronic Obstructive Lung Disease (GOLD) criteria [35]: Group 1 (G1) FEV1\% < 80\%; Group 2 (G2) FEV1\% $\geq 80 \%$ ). The threshold value coincided with median FEV1\% of population, making the two groups equal in terms of number of patients.

There was no difference in gender, age, pathologic stage, comorbidities and histology between the two groups, nor in incidence of major complications ( $15 \% \mathrm{Vs} 12 \%-\mathrm{p}=0.72)$ and postoperative ECOG status Considering the whole population (96 patients) which this series of patients came from, overall perioperative and in-hospital death was $6.5 \%$ in Group 1 and $11.5 \%$ in Group 2 ( $\mathrm{p}=0.35)$.

Comparing preoperative and postoperative PFT values in both groups, $\mathrm{FVC} \%$ and $\mathrm{RV} \%$, showed significant variations. In G2 a significant loss in DLCO was registered. Interestingly the FEV1\% loss was statistically significant only in G2 (88.3 to 73.7, p<0.001): in G1 FEV1\% loss was much less remarkable, decreasing from a mean value of 67.8 to 59.1 ( $p=n . s$.).

Variation of FEV1\% between the two groups after surgery was $-7.9 \%$ in Group 1 and $-14.9 \%$ in Group 2, showing a tendency to significance ( $\mathrm{p}=0.17$ ); also differences in $\mathrm{FVC} \%$ between the two groups were not remarkable $(-13.3 \%$ in Group 1 and $-6.7 \%$ in Group $2-p=0.33$ ). DLCO $\%$ loss was much higher in G2 than in G1 (-22.5 Vs $+1.5, \mathrm{p}=0.001)$. 
Six patients showed an improvement of actual postoperative FEV1\%, ranging from +1.5 to +7.3 compared with the preoperative value. All of these patients had a preoperative FEV1\% lower than $60 \%$, a homogeneous or upper lobe pattern emphysema as confirmed by both CT-scan and/or lung perfusion scintigraphy (when available) and had undergone a upper lobectomy. Type of lobectomy had no influence in FEV1\% loss in G2 (upper lobectomy: $14.5 \%$ - lower lobectomy: $-15.6 \%, \mathrm{p}=0.81$ ), as it demonstrated to have in G1 (upper lobectomy: $+5.4 \%$ - lower lobectomy: $-14.5 \%, \mathrm{p}=0.05$ ). (Figure 2 ).

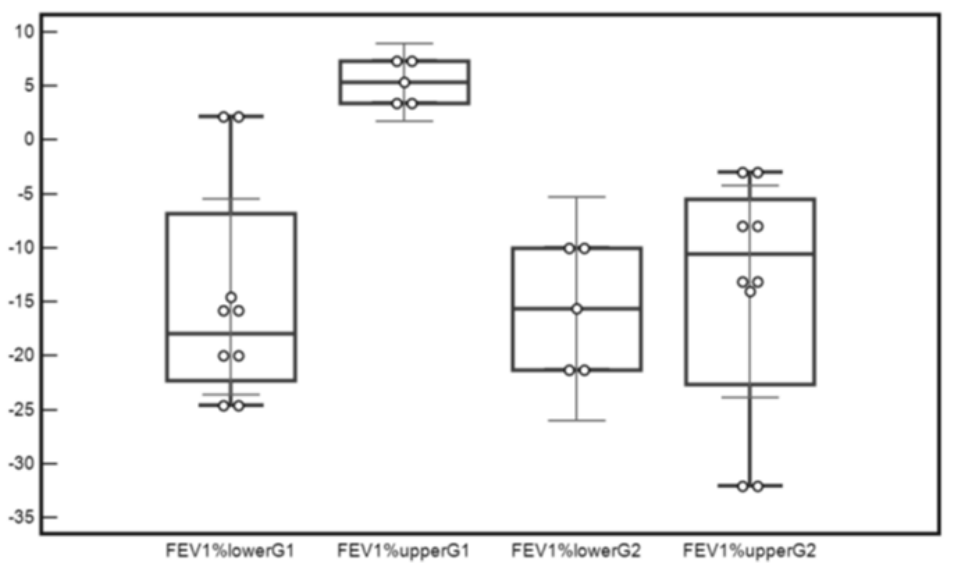

Figure 2. Variations of FEV1\% after surgery in the two groups (mean, standard deviation, range), stratified for type of resection (upper Vs lower lobe)

Considering FEV1 as a continuous variable, linear regression analysis showed a significant inverse correlations between preoperative value of FEV1\% and FEV1 percentage loss after surgery (B: $-0.63, R^{2}: 0.46-p<0.01$ ), even though linear correlation does not represent effectively the complex physiopathology following lung resection in patients with COPD/emphysema.

Blood gas tests reflected in some way the variations of PFT parameters: in G1 the lower loss in respiratory function resulted in not significant difference in $\mathrm{PaO} 2, \mathrm{PCO} 2$ and $\mathrm{pH}$ before and after surgery. Conversely patients in G2 showed a significant $\mathrm{PaO} 2$ decrease after surgery. No differences were found between the two groups at time of follow-up.

\subsection{Interpretation of results: does LVRS effect act also in octogenarians?}

Patients of this series obviously did not accomplish completely the criteria for LVRS and on the other hand apical wedge resection (and not lobectomy) is the recommended surgical option for lung volume reduction. Lobectomy implies removal of functioning lung tissue, together with emphysematous one, and this can explain the significant FVC\% loss in G1: this is not observed after LVRS performed in properly selected candidates. However, some physiological effects of LVRS remain and are responsible of the remarkable drop of RV\% 
and the not significant loss in FEV\% after surgery in G1: DLCO showed a gain of +1.5 at follow-up, probably due to more correct redistribution of ventilation and perfusion in lung zones. Patients in G2 having better pulmonary function, had the major impairment (of FEV1\% and also DLCO\%), because of the resection of a major portion of functioning lung. This results confirmed previous reports [36].

It must be noted that most of these studies report functional results after 1, 3 or 6 months: at this time, the post-thoracotomy pain could be still present in some cases, maybe causing a restrictive pattern and consequently a bias of the results [37]. In our series, all patients received a postero-lateral muscle-sparing thoracotomy, reducing a possible factor conditioning the amount of thoracic pain.

The functional advantage after an upper lobectomy than a lower lobectomy in patients with higher airflow impairment (G1) probably simply reflects predominantly upper lobe emphysema (as usual observed); however the same physiopatologic considerations should be valid in any case of resection of more emphysematous - less functioning lung lobe although no data can be provided supporting this consideration.

It should be kept in mind that PFTs are conditioned by patients collaboration and represent a kind of "surrogate" of effort tolerance which do not necessarily correlate with values measured at a single time. However postoperative global performance status and exercise tolerance seemed to reach a similar level in both mild and moderate COPD patients (G1 and G2), supporting the conclusion that differences in functional status are levelled after a major lung resection.

Although some limitations due retrospective nature of the study, and possible presence of bias such as the preoperative optimization of respiratory reserve through medications and/or physical rehabilitation, we tried to select an homogeneous cohort of patients with strict inclusion criteria in order to establish the pure effect of lobectomy after a long time, enough to allow remodelling processes of lung and thoracic wall until the final steady result. The inadequacy of sample size often did not allow to reach statistical significance in some tests. Lung perfusion scintigraphy was not routinely performed, so we had to use CTscan estimation method as surrogate to obtain corrected ppoFEV1.

Finally the retrospective nature of the study itself implies that some patients were denied surgery resulting, for example, in unavailability of data for patients with lower preoperative FEV1 and homogeneous pattern of emphysema undergone to lower lobectomy, so the benefit of upper lobectomy could be deducted only on physiological basis.

\section{Surgery - RT}

Since chemotherapy is not well tolerated by elderly people and yields very disappointing results in local control of disease, radiation therapy (RT) has ever been considered the most feasible and valuable therapeutic option in patients not fitting criteria for operability. 
Consequently, literature reports several series of patients treated with RT. In an old report dated 1988, results on 50 patients not candidate to surgery with peripherally located stage I NSCLC were analyzed: the overall response rate was $90 \%$, with $50 \%$ complete responses in tumors smaller than $4 \mathrm{~cm}$, resulting in overall survival rates of $56 \%$ at 2 years and $16 \%$ at 5 years (median survival of 27 months). Complete responders (20/50) showed a remarkable 5years survival of $42 \%$ with $25 \%$ of local relapse of disease. On the contrary none of the patients having a tumor greater than $4 \mathrm{~cm}$ was alive at 5 years. Results were favorable compared to those of a group of 70 patients surgically resected (median survival of 23 months).

The same positive results were reported several years later in a larger series of 347 patients: curiously, better results were achieved in elderly people (aged $>70$ ), although the difference was not significant, in terms of crude survival and disease-free survival [38]. Other Authors reported worse results of RT compared to surgery, with 5-year survival rate of only 10 to $20 \%$ after curative intent radiotherapy [39].

Unfortunately, very few studies report results of surgery and RT in the same Institution, most of them being retrospective and non-randomized.

Yendamuri compared overall survival after surgery (wedge resection) and three dimensional conformation radiation therapy in a retrospective study on 160 patients with early stage lung malignances unable to tolerate lobectomy. Propensity score-matched analysis and cost assessments were performed to compare outcomes with both modalities. Propensity matching was also performed to assure homogeneity between arms in terms of gender, histology, tumor size, performance status, and age The most frequent long term complications encountered were post-thoracotomy pain syndrome and radiation pneumonia with pulmonary fibrosis. . Surgery arm showed a trend to improved outcome towards radiotherapy one (57,1 months Vs 40,9 months), while mean cost of radiation therapy $(\$ 32,735)$ was not statistically significantly different from surgery $(\$ 30,411)$. Propensity matched analysis however failed to confirm a clear benefit of sublobar pulmonary resections, and it could be explained by inadequacy of number of patients and other bias (retrospective - not randomized trial, increased comorbidities of patients treated with 3-D conformal radiation) [40].

In another smaller series of compromised patients (not suitable for lobectomy) retrospectively reviewed (17 treated by sublobar resections, either segmentectomy or wedge resection and 18 by radiation therapy alone) overall 5 -years survival rates were $55 \%$ after surgery and $14,4 \%$ after radiation therapy $(p=0.004)$, without significant more complications due to lung resections. The advantage in long-term survival was maintained even considering only total or partial responders among patients treated with radiation therapy $(+18.8 \%$, $\mathrm{p}=0.008)$. Even in this series complications reported did not differ between the two treatment modalities (11.8 compared to 11.1). Local recurrence at margins of resection occurred in 4 patients in which tumour diameter was greater than $2 \mathrm{~cm}$. Authors concluded that surgery carries advantage over radiotherapy, especially for smaller lesions measuring less than $2 \mathrm{~cm}[41]$. 
A prospective analysis of QOL after radical radiotherapy noted benefit on haemoptysis, pain and anorexia, while cough, dyspnea and fatigue were poorly alleviated; also global QOL responded poorly [42].

\section{Novel perspective for management of lung cancer in octogenarians}

\subsection{Diagnosis - Staging}

Histological proven diagnosis is significant less frequent in elderly: this may reflect the poor perceived fitness of withstand bronchoscopy, CT guided biopsy and other invasive techniques [43].

Preoperative histopatological confirmation should preferably be obtained in elderly people to avoid unnecessary surgery for benign lesions or, to identify patients with poor prognosis related histology (small-cell carcinoma, large-cell undifferentiated neuroendocrine carcinoma).

Trans-thoracic Ultrasound or CT guided Fine Needle Aspiration was found to have equivalent safety and tolerance to procedure in elderly patients (aged 70 or more), with majority of cases performed as a day-case [44].

In our series of patients, concordance of clinical and pathological staging was a not exciting $63 \%$ : the use of sole CT-scan and blind transbronchial/trans-tracheal endoscopic fine needle biopsy has certainly played a role in understaging. The introduction of PET-CT scan in routine assessment for staging purpose in most Institution demonstrated to enhance conventional staging, both for nodal status and distant metastasis, resulting in a $10 \%$ of downstaging and $33 \%$ upstaging. This changed intent of treatment from palliative to curative in $4 \%$ and vice-versa in $22 \%$ of cases [45].

Endoscopic UltraSound guided Fine Needle Aspiration (EUS-FNA) performed by bronchoscopy by skilled operators promises to improve sensitivity in metastasis detection of PET positive lymph-nodes not necessarily increased in size (CT-scan nodal assessment is essentially based on dimensional criteria and post-contrast perfusion nodal pattern and is operator-dependent).

A prospective study on 86 with mediastinal adenopathies provided impressive negative and positive predictive value ( $94 \%$ and $100 \%$ respectively). This allowed to shift to non-surgical management in $80 \%$ of cases [46].

\subsection{Image Guided Radiotherapy/Intensity Modulated Radiation Therapy (IGRT/IMRT)}

This novel techniques promise to be a milestone in radiation treatment for capacity to delineate tumour contour and correct it for patients positioning, resulting in two theoretical high advantages compared to traditional radiation therapy. 
1. Greater precision in irradiating tumour volume with minimal toxicity of neighbouring healthy tissues ( $\rightarrow$ lower incidence of side effects)

2. Possibility to deliver higher dosage with presumed higher efficacy

Unfortunately, despite this convincing rationale, literature lacks of robust studies to confirm promising clinical benefits. To date, there is enough evidence on technical performance, encouraging but not yet conclusive information on safety, very weak evidence in clinical effectiveness and none on cost-effectiveness. The proportion of patients with the clinical indications for IGRT/IMRT inferred by the RER (Regione Emilia Romagna) regional database result in an estimate $10 \%$ of all lung cancers (the lowest percentage among several solid tumours considered - brain, prostate, head and neck, pancreatic).

Nonetheless IGT/IMRT are worthy to be seriously considered in future clinical trials, preferably involving a surgery arm.

\subsection{Minimally invasive surgery: VATS lobectomy}

Videothoracoscopic Assisted Thoracic Surgery has in recent years gained again popularity and has become of routinely use in some Institutions for early stages lung cancers not involving fissures of hilar elements. Octogenarians seem to have the proper characteristics for indication to VATS major resections. Firstly, as stated above, only patients with stage I-II tumours amenable to be resected by standard lobectomy should be operated on; secondly, advantages of VATS compared to thoracotomy (less postoperative pain, lenght of stay, functional impairment) are theoretically able to reduce complications and mortality in such frail patients.

A recent study on 95 octogenarians who received surgery either by VATS $(n=58)$ or standard thoracotomy $(\mathrm{n}=37)$ in a single Institution, demonstrated that major cardiopulmonary complications occurred in $13.8 \%$ of VATS group and $32.4 \%$ of open surgery group $(p=0.03$ ). Five-years survival was not affected but study was retrospective, non-randomized and suffers some bias in patients selection (more I stages in VATS group) [47]. However efficacy of VATS has been reported by several other Authors [48, 49]. It has been demonstrated that VATS surgery generates less cytokine production in early postoperative phase and less impairment of postoperative pulmonary function, which ultimately could decrease inhospital complications, as stated in the text.

Therefore there is enough evidence to support minimally invasive surgery especially in very elderly people.

\subsection{Thermal ablation}

Thermal ablation has been shown to be effective as local treatment of solid tumours. The main modalities to achieve thermal damage of malignant tissue are radiofrequency (RF), microwave (MW) and cryoablation; all them are applied directly to tumour through different devices positioned percutaneously through an image-guided procedure. 
Lung tissue has unique characteristics which maximizes the so called heat sink effect: airflow and bloodflow limits in fact the intended tissue damage, that results smaller in vivo versus in vitro experiments, acting as an air/liquid-cooled radiator. Lung has therefore a small thermal conductivity.

In these regards MW has some theoretical advantages over RF ablation, because thermal damage is realized through electromagnetic waves that cause polar water molecules to rotate rapidly; MW is not limited by thermal and electric conductivity of tissue, reaching so larger zones and higher temperatures in lung [50]. Histopathological studies indicates that a margin of $8 \mathrm{~mm}$ for adenocarcinomas and $6 \mathrm{~mm}$ for squamous cell carcinoma are necessary to cover $95 \%$ of microscopic disease [51].

Thermal ablation techniques presents similar limits regarding to:

- The lack of pathological data, especially histological and molecular features of tumour, nodal status and margins of treatment: regarding the last issue, cryoablation is more attractive because the ablated zone correlates with the ice-ball image visualized by CTscan; this imply nonoptimal planning of adjuvant therapies

- Local control of disease: tumours greater than $3 \mathrm{~cm}$ in diameter have a low likelihood of successful ablation with RF; MW ablation has shown promising rates of local control also for tumours until $5 \mathrm{~cm}$; overall local tumour progression rate was reported to be $50 \%$ after RF for c-I NSCLC, compared to $28.6 \%$ after surgery [52]; some studies indicate that combination of thermal ablation (more effective in the centre of tumour) and external beam radiotherapy (more effective at periphery of the lesion) could remarkably improve local control of disease [53]

- Evaluation of treatment response and monitoring of recurrences is more challenging after thermal ablation, because both residual mass and ablation zone are present: inflammatory response to heat damage may be responsible of initial apparent increase in size of tumour; FDG uptake at PET scan, in addition to CT criteria, is useful to predict completeness of tumour ablation but it should not be performed before 3 months to prevent false positives due to inflammatory response [54]

- Complications: major complication (pneumothorax requiring chest tube drainage, air embolism and pulmonary abscess) are reported in about $8 \%$ of procedures, depending on tumour size and pulmonary reserve; minor complications as hemoptysis, fever, pleuritis with effusion, damages to phrenic or intercostals nerves are much more frequent (cumulative incidence: $50 \%$ ) and may sometimes significantly impair quality of life of patients with poor baseline performance status [50]

Regarding the last point, Tada and coll. reported that pulmonary function significantly decreased after RF, with FEV1 and VC at 1 and 3 months significantly lower than the baseline: predictive factor of major functional loss were severe pleuritis (at 1 and 3 month) and ablation of large volume of marginal parenchyma (at 3 months). Shrinkage of ablated zone during healing process may retract surrounding lung tissue, resulting in a decrease of alveoli compliance [55]. 
Indications to RF should therefore adequately determined for severely impaired patients as well as indications to surgery.

A recent review based on medically inoperable, early-stage NSCLC patients reports an advantage in 5-years survival and local recurrence rates of patients treated with stereotactic RT versus those treated by $\mathrm{RF}$, at the cost of more disabling and lasting complications (actinic pneumonia, fatigue) [56].

We can conclude that thermal ablation should be part of the armamentarium against lung cancer in a multidisciplinary setting: its undeniable advantages (single session treatment, feasibility in marginal patients, repeatability) make it not only an alternative to surgery (e.g.: multiple small nodules) but primarily a complementary treatment (e.g.: treatment of recurrences after surgery, persistence of local disease after RT).

\section{Take home messages}

Provided that marginal patients should be evaluated in multi-disciplinary setting and on individual basis, some concepts should be kept in mind in management of octogenarians affected by NSCLC.

- Age above than 80 is neither a contraindication to operative treatment nor a routine indication for lesser resection; even octogenarians, with reduced life expectancy, dead of cancer rather than of comorbidities

- The presence of comorbidities (cardio-respiratory in particular) predict increased operative risk. This support the hypothesis that the potential association between advancing age and operative mortality is a reflection of increased co-morbidity rather than age per se.

- Even healthy octogenarians could have reduced organ function, resulting in low tolerance of postoperative major complications, which are fatal in a considerable percentages of patients.

- Extended resections should be avoided in octogenarians: lesions in early stages and yet not resectable with a standard lobectomy should therefore preferably managed in nonoperative way.

- Complete and accurate clinical staging is crucial since pathological stage is the only predictive factor affecting long term survival. Accurate preoperative clinical staging is imperative and in this respect, a more standard use of PET-TC appear to be necessary. In case of doubt, invasive staging with mediastinoscopy should be used in the process of selection of candidates for surgery to avoid unnecessary operative risk in poor prognosis patients.

- $\quad$ Surgery remains to date the benchmark in terms of local control of disease and should therefore be preferred to radiation therapy and thermal ablation until future studies will eventually provide evidences of better risk/benefit ratio. Minimally invasive surgery promises to carry a benefit on postoperative outcome of elderly people 
- Lung volume reduction criteria applied to lung surgery could move the limit of operability: in case of poor respiratory reserve, additional considerations should be made before excluding them from surgery, because ppoFEV1\% is inaccurate in particular cases. These more compromised patients could benefit of a preoperative pulmonary rehabilitation program: in this way it could be possible to minimize the rate of minor postoperative complications that are probably related to 1st day FEV1\% [57]. Lung volumes remodelling in fact takes at least some months to complete and favourable effects are not immediately available.

The critical issue is then to define which octogenarians will have the best chance of avoiding complications and ultimately benefit in terms of long survival after pulmonary resection and which are medically inoperable.

\section{Conclusion}

Octogenarians with adequate organ function affected by potentially resectable lung cancer are worthy of curative treatments because life expectancy almost always exceed prognosis of disease. Octogenarian should be considered as a patient with higher possibility to have multiple comorbidities and or reduced organ function (even subclinical) and should have access to lung cancer services regardless of age. High accuracy is recommended in clinical staging and functional assessment in order to select healthy patients who could really benefit of lung surgery with acceptable operative risk and with acceptable postoperative QOL. Surgical therapy is nowadays well accepted for healthy octogenarians. In marginal patients, appropriate counselling can support physician to decide on case-by-case basis whether to favour QOL over survival or vice-versa (as usually in the younger patients). Such patients should ideally be enrolled in randomised trials to provide an evidence base.

\section{Author details}

Cristian Rapicetta, Massimiliano Paci, Tommaso Ricchetti, Sara Tenconi and Giorgio Sgarbi

Thoracic Surgery Unit, Arcispedale Santa Maria Nuova - Istituto di Ricerca e Cura a Carattere Scientifico, Reggio Emilia, Italy

Salvatore De Franco

Medical Education - Health Innovation Unit, Arcispedale Santa Maria Nuova - Istituto di Ricerca e Cura a Carattere Scientifico, Reggio Emilia, Italy

\section{References}

[1] Ginsberg RJ, Hill LD, Eagan RT, et al. Modern thirty-day operative mortality for surgical resections in lung cancer. J Thorac Cardiovasc Surg 1983;86:654-658. 
[2] Romano PS, Mark DH. Patients and hospital characteristics related to in-hospital mortality after lung cancer resection. Chest 1992;101:1332-1337.

[3] Smith TJ, Penberthy L, Desch CE, Whittemore M, Newschaffer C, Hillner BE, McClish $\mathrm{D}$, Retchin SM. Differences in initial treatment patterns and outcomes of lung cancer in the elderly. Lung Cancer 1995;13:235-252.

[4] Janssen-Heijnen MLG, Smulders S, Lemmens VEPP, et al. Effect of comorbidity on the treatment and prognosis of elderly patients with non-small cell lung cancer. Thorax 2004;59:602-607.

[5] National Cancer Intitute. SEER statistics review 1973-1997. Bethesda, MD: National Cancer Institute, 2000.

[6] Voltolini L, Rapicetta C, Ligabue T, Luzzi L, Scala V, Gotti G. Short and long-term results of lung resection for cancer in octogenarians. Asian Cardiovasc Thorac Ann 2009;17(2):147-52.

[7] Pagni S, Federico JA, Ponn RB. Pulmonary resection for lung cancer in octagenarians. Ann Thorac Surg 1997;63:785-789.

[8] Port JL, Kent M, Korst RJ, Lee PC, Levin MA, Flieder D, Altorki NK. Surgical resection for lung cancer in the octogenarian. Chest 2004;126:733-738.

[9] Aoki T, Yamato Y, Tsuchida M, Watanabe T, Hayashi J, Hirono T. Pulmonary complications after surgical treatment of lung cancer in octogenarians. Eur J Cardiothorac Surg 2000;18:662-665.

[10] Brock MV, Kim MP, Hooker CM, Alberg AJ, Jordan MM, Roig CM, Xu L, Yang SC. Pulmonary resection in octogenarians with stage I non-small cell lung cancer: a 22 year experience. Ann Thorac Surg 2004;77:271-277.

[11] Harvey JC, Erdman C, Pisch J, Beattie EJ. Surgical treatment of non-small cell lung cancer in patients older than seventy years. J Surg Oncol 1995;60:247-9.

[12] Naunheim KS, Kesler KA, D'Orazio SA, et al. Lung cancer surgery in the octogenarian. Eur J Cardiothorac Surg. 1994;8:453-6.

[13] Mountain CF. Revision in the International System for staging lung cancer. Chest 1997;111:1710-1717.

[14] Sirbu H, Schreiner W, Dalichau H, Busch T. Surgery for non-small cell carcinoma in geriatric patients: 15-year experience. Asian Cardiovasc Thorac Ann. 2005;13(4):3306.

[15] Sioris T, Salo J, Perhoniemi V, Mattila S. Surgery for lung cancer in the elderly. Scand Cardiovasc J. 1999;33(4):222-7.

[16] Au J, el-Oakley R, Cameron EW. Pneumonectomy for bronchogenic carcinoma in the elderly. Eur J Cardiothorac Surg 1994;8:247-250.

[17] British Thoracic Society, Society of cardiothoracic Surgeons of Great Britain, and Ireland Working Party. Guidelines on the selection of patients with lung cancer for surgery. Thorax 2001;56:89-108.

[18] Thomas P, Sielezneff I, Ragni J, Giudicelli R, Fuentes P. Is lung cancer resection justified in patients aged over 70 years? Eur J Cardiothorac Surg.1993;7:241-245.

[19] Miller DL, Orszulak TA, Pairolero PC, Trastek VF, Schaff HV. Combined operation for lung cancer and cardiac disease. Ann Thorac Surg. 1994 Oct;58(4):989-93. 
[20] Dominguez-Ventura A, Allen MS, Cassivi SD, Nichols FC 3rd, Deschamps C, Pairolero PC. Lung cancer in octogenarians: factors affecting morbidity and mortality after pulmonary resection. Ann Thorac Surg 2006;82:1175-9.

[21] Suemitsu R, Yamaguchi M, Takeo S, Ondo K, Ueda H, Yoshino I, Maehara Y. Favorable surgical results for patients with nonsmall cell lung cancer over 80 years old: a multicenter survey. Ann Thorac Cardiovasc Surg. 2008;14(3):154-60.

[22] Fanucchi O, Ambrogi MC, Dini P, Lucchi M, Melfi F, Davini F, Mussi A. Surgical treatment of non-small cell lung cancer in octogenarians. Interact Cardiovasc Thorac Surg. 2011;12(5):749-53.

[23] Brantigan OC. Surgical treatment of pulmonary emphysema. Maryland State Med J 6:409, 1957.

[24] DeMeester SR, et al. Lobectomy combined with volume reduction for patients with lung cancer and advanced emphysema. J Thorac Cardiovasc Surg 1998;115:681,.

[25] DeRose JJ, et al: Lung reduction operation and resection of pulmonary nodules in patients with severe emphysema. Ann Thorac Surg 1998;65:314.

[26] Kearney DJ, Lee TH, Reilly JJ, DeCamp MM, Sugarbaker DJ. Assessment of operative risk in patients undergoing lung resection. Importance of predicted pulmonary function. Chest. 1994;105(3):753-9.

[27] Yoshimoto K, Nomori H. Prediction of pulmonary function after lung lobectomy by subsegments counting, computed tomography, single photon emission computed tomography and computed tomography: a comparative study. Eur J Cardiothorac Surg. 2009;35(3):408-13.

[28] McKenna RJ Jr, Brenner M, Fischel RJ, Singh N, Yoong B, Gelb AF, Osann KE. Patient selection criteria for lung volume reduction surgery. J Thorac Cardiovasc Surg. 1997 Dec;114(6):957-64; discussion 964-7.

[29] Schattenberg T, Muley T, Dienemann H, Pfannschmidt J. Impact on pulmonary function after lobectomy in patients with chronic obstructive pulmonary disease. Thorac Cardiovasc Surg 2007;55: 500-504.

[30] Liao W, Ma G, Fang Y, Wang CM. Influence of chronic obstructive pulmonary disease on postoperative lung function of lung cancer patients and predictive value of lung perfusion scan. Ai Zheng. 2009;28(6):642-6.

[31] Santambrogio L, Nosotti M, Baisi A, Ronzoni G, Bellaviti N, Rosso L. Pulmonary lobectomy for lung cancer: a prospective study to compare patients with forced expiratory volume in 1 second less than $80 \%$ of predicted. Eur Cardiothorac Surg 2001; 20:684-687.

[32] Luzzi L, Tenconi S, Voltolini L, Paladini P, Ghiribelli C, Di Bisceglie M, Gotti G. Long term respiratory functional results after pneumonectomy. Eur J Cardiothorac Surg 2008;34(1):164-8.

[33] Rapicetta C, Tenconi S, Voltolini L, Luzzi L. Impact of lobectomy for non-small-cell lung cancer on respiratory function in octogenarian patients with mild to moderate chronic obstructive pulmonary disease. Eur J Cardiothorac Surg. 2011;39(4):555-9.

[34] Goddard PR, Nicholson EM, Laszo G, Watt I. Computed tomography in pulmonary emphysema. Clin Radiol 1982; 33:379-387. 
[35] Glaab T, Banik N, Rutscmann OT, Wenker M. National survey of guideline-compliant COPD management among pneumologist and primary care physicians. COPD 2006;3(3):141-8.

[36] Marchand E, Gayan-Ramirez G, De Leyn P, Decramer M. Physiological basis of improvement after lung volume reduction surgery for severe emphysema: where are we? Comment in: Eur Respir J. 1999;13(3):480-1.

[37] Sabanathan S, Eng J, Mearns AJ. Alterations in respiratory mechanics following thoracotomy. J R Coll Surg Edinb. 1990 Jun;35(3):144-50. Review.

[38] Gauden SJ, Tripcony L. The curative treatment by radiation therapy alone of Stage I non-small cell lung cancer in a geriatric population. Lung Cancer. 2001 Apr;32(1):71-9.

[39] Sibley GS, Jamieson TA, Marks LB, Anscher MS, Prosnitz LR. Radiotherapy alone for medically inoperable stage I non-small cell lung cancer: the Duke experience. Int J Radiat Oncol Biol Phys 1998;40:149-54.

[40] Yendamuri S, Komaki R, Correa AM, Allen P et al. Comparison of limited surgery and three-dimensional conformal radiation in high-risk patients with stage I non-small cell lung cancer. J Thorac Oncol 2007; 2: 1022-1028.

[41] Yano T, Yokoyama H, Yoshino I, Tayama K, Asoh H, Hata K, Ichinose Y. Results of limited resection for compromised or poor-risk patients with clinical stage I non-small cell lung carcinoma of the lung. J Am Coll Surg 1995; 181: 33-37.

[42] Langendijk JA, Aaronson NK, de Jong JM, ten Velde GP, Muller MJ, Lamers RJ, Slotman BJ, Wouters EF. Prospective study on quality of life before and after radical radiotherapy in non-small-cell lung cancer. J Clin Oncol. 2001;15;19(8):2123-33.

[43] Booton R, Jones M, Thatcher N. Lung cancer 7: management of lung cancer in elderly patients. Thorax. 2003;58(8):711-20.

[44] Brown TS, Kanthapillai P. Transthoracic needle biopsy for suspected thoracic malignancy in elderly patients using CT guidance. Clin Radiol. 1998;53(2):116-9.

[45] Hicks RJ, Kalff V, MacManus MP, Ware RE, Hogg A, McKenzie AF, Matthews JP, Ball DL. (18)F-FDG PET provides high-impact and powerful prognostic stratification in staging newly diagnosed non-small cell lung cancer. J Nucl Med. 2001;42(11):1596604.

[46] Wiersema MJ, Vazquez-Sequeiros E, Wiersema LM. Evaluation of mediastinal lymphadenopathy with endoscopic US-guided fine-needle aspiration biopsy. Radiology. 2001;219(1):252-7.

[47] Igai H, Takahashi M, Ohata K, Yamashina A, Matsuoka T, Kameyama K, Nakagawa $\mathrm{T}$ et al. Surgical treatment for non-small cell lung cancer in octogenarians: the usefulness of video-assisted thoracic surgery. Interact Cardiovasc Thorac Surg. 2009;9(2):274-6.

[48] Mun M, Kohno T. Video-assisted thoracic surgery for clinical stage I lung cancer in octogenarians. Ann Thorac Surg. 2008;85(2):406-11.

[49] Cattaneo SM, Park BJ, Wilton AS, Seshan VE, Bains MS, Downey RJ, Flores RM, Rizk N, Rusch VW. Use of video-assisted thoracic surgery for lobectomy in the elderly results in fewer complications. Ann Thorac Surg. 2008;85(1):231-5. 
[50] Sonntag PD, Hinshaw JL, Lubner MG, Brace CL, Lee FT Jr. Thermal ablation of lung tumors. Surg Oncol Clin N Am. 2011;20(2):369-87.

[51] Ambrogi MC, Fontanini G, Cioni R, Faviana P, Fanucchi O, Mussi A. Biologic effects of radiofrequency thermal ablation on non-small cell lung cancer: results of a pilot study. $\mathrm{J}$ Thorac Cardiovasc Surg. 2006 May;131(5):1002-6.

[52] Kim SR, Han HJ, Park SJ, Min KH, Lee MH, Chung CR, Kim MH, Jin GY, Lee YC. Comparison between surgery and radiofrequency ablation for stage I non-small cell lung cancer. Eur J Radiol. 2012 Feb;81(2):395-9. doi:10.1016/j.ejrad.2010.12.091.

[53] Grieco CA, Simon CJ, Mayo-Smith WW et al. Percutaneous image-guided thermal ablation and radiation therapy: outcomes of combined treatment for 41 patients with inoperable stage I/II non-small-cell lung cancer. J Vasc Interv Radiol. 2006;17(7):1117-24.

[54] Deandreis D, Leboulleux S, Dromain C, Auperin A, Coulot J, Lumbroso J, Deschamps F, Rao P, Schlumberger M, de Baère T. Role of FDG PET/CT and chest CT in the follow-up of lung lesions treated with radiofrequency ablation. Radiology. 2011;258(1):270-6.

[55] Tada A, Hiraki T, Iguchi T, Gobara H, Mimura H, Toyooka S, Kiura K, Tsuda T, Mitsuhashi T, Kanazawa S. Influence of Radiofrequency Ablation of Lung Cancer on Pulmonary Function. Cardiovasc Intervent Radiol. 2011 Jul 2. [DOI: 10.1007/s00270-0110221-z].

[56] Bilal H, Mahmood $S$ et al. Is radiofrequency ablation more effective than stereotactic ablative radiotherapy in patients with early stage medically inoperable nonsmall cell lung cancer? Interact Cardiovasc Thorac Surg. 2012 May 10. doi: 10.1093/icvts/ivs1.

[57] Varela G, Brunelli A, Rocco G, Novan N, Refai M, Jimenez MF, Salati M et al .Measured FEV1 in the first postoperative day, and not ppoFEV1, is the best predictor of cardio-respiratory morbidity after lung resection. Eur J Cardiothorac Surg 2007;32(5):783-6.

[58] Wu MT, Chang JM, Chiang AA, Lu JY, Hsu HK, Hsu WH, Yang CF. Use of quantitative $\mathrm{CT}$ to predict postoperative lung function in patients with lung cancer. Radiology 1994;191:257-262.

[59] Noordijk E, Poest C, Hermans J et al: Radiotherapy as an alternative to surgery in elderly patients with respectable lung cancer. Radiother Oncol 1988; 13:83.

[60] Janssen F, Kunst A. The choice among past trends as a basis for the prediction of future trends in old-age mortality. Popul Stud (Camb). 2007;61(3):315-26.

[61] Korst RJ, Ginsberg RJ, Ailawadi M, Bains MS, Downey RJ Jr, Rusch VW, Stover D. Lobectomy improves ventilatory function in selected patients with severe COPD. Ann Thorac Surg 1998;66:898.

[62] Volpino P et al. Risk of mortality from cardiovascular and respiratory causes in patients with chronic obstructive pulmonary disease submitted to follow-up after lung resection for non-small cell lung cancer. J Cardiovasc Surg (Torino). 2007;48(3):37583. 
[63] Ambrogi MC, Lucchi M, Dini P, Melfi F, Fontanini G, Faviana P, Fanucchi O, Mussi A. Percutaneous radiofrequency ablation of lung tumours: results in the mid-term. Eur J Cardiothorac Surg. 2006;30(1):177-83. 